\begin{abstract}
UNIVERSIDADE DE SÃO PAULO
FACULDADE DE MEDICINA DE RIBEIRÃO PRETO

DEPARTAMENTO DE GINECOLOGIA E OBSTETRÍCIA
\end{abstract}

Ecocardiografia fetal no primeiro trimestre da gestação

Sandra Regina Marques Carvalho

Ribeirão Preto - São Paulo 
Sandra Regina Marques Carvalho

\section{Ecocardiografia fetal no primeiro trimestre da gestação}

Dissertação de Mestrado, apresentada ao

Programa de Ginecologia e Obstetrícia do

Curso de Pós Graduação da FMRP - USP.

Área de Concentração: Ginecologia e Obstetrícia

Orientador: Aderson Tadeu Berezowski

Ribeirão Preto - São Paulo 
AUTORIZO A REPRODUÇÃO E DIVULGAÇÃO TOTAL OU PARCIAL DESTE

TRABALHO, POR QUALQUER MEIO CONVENCIONAL OU ELETRÔNICO, PARA FINS DE ESTUDO E PESQUISA, DESDE QUE CITADA A FONTE.

Carvalho, Sandra Regina Marques

Ecocardiografia fetal no primeiro trimestre da gestação. Ribeirão Preto, 2006.

44 p. : il. ; $30 \mathrm{~cm}$

Dissertação de Mestrado, apresentada à Faculdade de Medicina de Ribeirão Preto/USP. Área de concentração: Ginecologia e Obstetrícia.

Orientador: Berezowski, Aderson Tadeu.

1. Ecocardiografia fetal. 2. Primeiro trimestre. 3. Ultrasonografia transvaginal. 4. Diagnóstico pré-natal. 5. Gravidez normal. 


\section{DEDICATÓRIA}

Aos meus pais e a minha família pelo amor, compreensão, apoio e estímulo à minha formação pessoal e profissional. 


\section{AGRADECIMENTOS}

Ao Professor Dr.Sérgio Pereira Cunha, que permitiu meu ingresso no Departamento de Ginecologia e Obstetrícia do Hospital das Clínicas da Faculdade de Medicina de Ribeirão Preto.

Ao Prof. Dr. Aderson Tadeu Berezowski, pela amizade, incentivo e apoio sempre presente em todos os momentos, pela oportunidade de trabalhar com ecocardiografia na área de medicina fetal e pela orientação no desenvolvimento deste trabalho.

A Prof. Dra. Maria Célia Mendes, pela amizade, auxílio na aprendizagem do exame ultra-sonográfico obstétrico de primeiro trimestre, encaminhamento das pacientes para o meu estudo, e principalmente, pela amizade e pelo estímulo em todos os momentos.

Ao Prof. Dr. Omero Poli Neto, pela amizade, carinho e interesse na assessoria da análise estatística e orientação no desenvolvimento deste trabalho.

A todos os Professores do Departamento de Ginecologia e Obstetrícia .do Hospital das Clínicas da Faculdade de Medicina de Ribeirão Preto pela oportunidade de dar continuidade a minha formação profissional.

A todos, que de alguma forma, contribuíram para a minha formação e para o desenvolvimento deste trabalho. 


\section{SUMÁRIO}

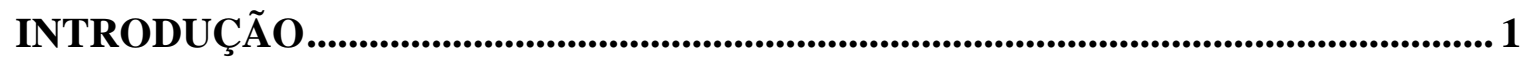

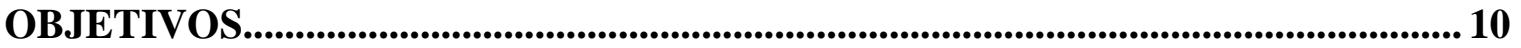

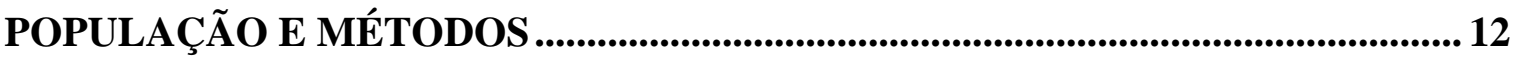

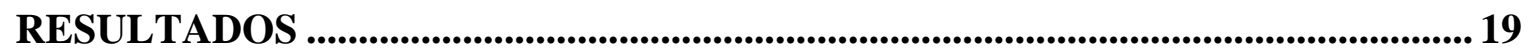

DISCUSSÃO

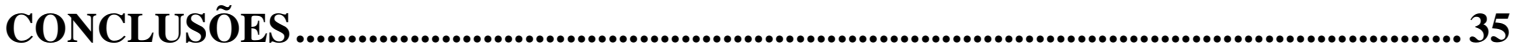

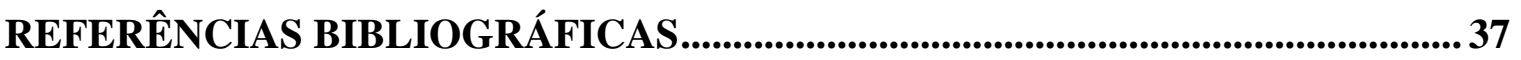




\section{LISTA DE ILUSTRAÇÕES}

Figura 1: Desenho esquemático dos cortes ecocardiográfico básicos utilizados, demonstrando como foram realizadas as medidas dos diâmetros

Figura 2: Imagens obtidas dos cortes ecocardiográficos básicos utilizados. A: Plano

quatro câmaras, B: Eixo longo, C: Eixo curto .........................................................18

Figura 3: Representação gráfica da relação entre os diâmetros dos anéis valvares mitral, tricúspide e o diâmetro do comprimento crânio-nádegas em milímetros ................23

Figura 4: Representação gráfica da relação entre os diâmetros dos anéis valvares aórtico e pulmonar e o diâmetro do comprimento crânio-nádegas em milímetros 


\section{RESUMO}

CARVALHO, S. R. M. Ecocardiografia fetal no primeiro trimestre da gestação. 2006.

Dissertação (Mestrado) - Faculdade de Medicina de Ribeirão Preto, Universidade de São Paulo.

Os objetivos deste estudo foram determinar o índice sucesso na realização de um ecocardiograma fetal completo entre a $11^{\mathrm{a}}$ e a $14^{\mathrm{a}}$ semanas, estabelecer a semana mais adequada para a realização de um exame completo, mensurar os diâmetros dos anéis das valvas cardíacas e comparar os diâmetros obtidos com o comprimento crânio-nádegas (CCN) correlacionando a evolução das dimensões cardíacas com o crescimento fetal nesta fase evolutiva.

Avaliamos por ecocardiografia 46 fetos que apresentavam translucência nucal e avaliação Doppler do fluxo do ducto venoso normais. O estudo ecocardiográfico fetal foi realizado por via transvaginal, entre a $11^{\mathrm{a}}$ e a $14^{\mathrm{a}}$ semanas, com intervalos seriados de uma semana. Em cada exame, foram obtidos os três principais planos ecocardiográficos básicos e os diâmetros das valvas aórtica, pulmonar, mitral e tricúspide. As medidas dos diâmetros valvares foram comparadas ao diâmetro do CCN.

Os resultados demonstraram uma taxa de sucesso na obtenção de um ecocardiograma completo de $37 \%$ na $11^{\mathrm{a}}$ semana, de $85 \%$ na $12^{\mathrm{a}}$ semana e de $100 \%$ entre a $13^{\mathrm{a}}$ e a $14^{\mathrm{a}}$ semanas. O corte mais freqüentemente obtido, nas duas primeiras semanas, foi o eixo longo e o mais difícil de ser visibilizado foi o eixo curto. As curvas de crescimento dos diâmetros das valvas mitral, tricúspide, aórtica e pulmonar demonstraram uma correlação linear com o crescimento fetal com valores de $\mathrm{r}^{2}$ de 0,74, 0,75, 0,77 e 0,73. Não houve diferença 
estatisticamente significativa entre os diâmetros das valvas atrioventriculares e entre os diâmetros das valvas ventriculoarteriais.

A obtenção dos três planos ecocardiográficos básicos em $85 \%$ dos casos na $12^{\text {a }}$ semana e 100\% dos casos, a partir da $13^{\mathrm{a}}$ semana, demonstra que é possível a realização de um ecocardiograma fetal completo no primeiro trimeste da gestação, por via transvaginal. Houve correlação positiva entre os diâmetros valvares e o CCN demonstrando uma curva de crescimento neste período. A comparação entre diâmetros valvares mitral e tricúspide, do mesmo modo que, a comparação entre os diâmetros valvares aórtico e pulmonar não apresentaram diferenças estatisticamente significativas, sugerindo não haver dominância no tamanho destas estruturas, nesta fase do desenvolvimento em fetos normais.

Palavras-chave: Ecocardiografia Fetal, Primeiro Trimestre, Ultra-sonografia Transvaginal, Gravidez Normal, Diagnóstico Pré-natal. 
ABSTRACT 


\section{ABSTRACT}

\section{Carvalho, S.R.M. Fist trimester fetal echocardiography.}

The aim of this study was to assess the feasibility of performing a complete echocardiography study during the first trimester of pregnancy by transvaginal approach and to establish the best week to accomplish a complete evaluation. A correlation between the measurements of the diameters of the cardiac valves and the gestational age along this period of the fetal life was made. Forty-six fetuses with normal nuchal translucency and Doppler evaluation of the venous duct were submitted to fetal echocardiography studies by transvaginal approach between the 11 and 14 weeks of gestation with a week interval. The following measurements were obtained: mitral, tricuspid, aortic and pulmonary valves diameters and the crown-rump length (CRL). The obtained results of the cardiac dimensions were compared with the CRL in the four weeks determinations. The rates of complete echocardiography evaluation were $37 \%, 85 \%$ and $100 \%$ at 11,12 and $13-14$ weeks, respectively. The longitudinal view was the easiest to obtain (100\% at 12 weeks) and the short view was the most difficult one. There was no statistically significant difference between neither the diameters of the mitral and tricuspid nor the aortic and pulmonary valves. The diameters of the valves were correlated with the CRL and a linear growing curve was obtained with values of $r^{2}$ of $0.74,0.75,0.77$ and 0.73 respectively. The study demonstrated the feasibility of a complete fetal echocardiography evaluation by transvaginal approach during the first trimester of gestation. The rate of a complete evaluation increased along the period and reached $100 \%$ at 13 weeks. The longitudinal view was the easiest to obtain with a success rate of $100 \%$ at 12 week. There was a linear correlation between de cardiac valves diameters and the cranial caudal measurement revealing a relationship between the cardiac and fetal development during this phase of 
fetal life. The absence of statistically significant difference between the left and right valves dimensions possibly means that there is no predominance of right or left chambers during this period of evaluation.

KeyWords: Fetal Echocardiography, First Trimester, Transvaginal Ultrasound, Normal Pregnancies, Prenatal Diagnosis. 
INTRODUÇÃO 


\section{INTRODUÇÃO}

As cardiopatias congênitas são as malformações mais freqüentes ao nascimento apresentando incidência estimada entre 3,5 a 12 em cada mil recém-nascidos (HOFFMAN, 1995a).

As malformações cardíacas, quando ocorrem isoladamente, são seis vezes mais freqüentes que as anomalias cromossômicas e quatro vezes mais freqüentes que as anomalias do tubo neural, embora a associação entre cardiopatia congênita e anomalia cromossômica ou outras anomalias extracardíacas seja comum (SMALL; COPEL, 2004).

Considerando-se a elevada taxa de abortamento que ocorre no primeiro trimestre, em geral causada pela presença de anomalias cromossômicas e anomalias estruturais graves, estima-se que a incidência de cardiopatia congênita intra-útero seja até cinco vezes maior que no neonato.Não há, porém, estudos de necropsia, realizados nesta fase, que possam demonstrar a real incidência desses defeitos (HOFFMAN, 1995b).

O desenvolvimento anormal do coração ou grandes vasos ocorre, principalmente, nas primeiras oito semanas de gestação, período que corresponde a embriogênese cardíaca. Na maioria dos casos, não é possível determinar o(s) fator(es) teratogênicos incidente(s), apontando-se para uma etiologia multifatorial, onde vários fatores de risco (genéticos, ambientais, químicos e maternos) atuam de forma isolada ou associada. Os principais mecanismos patogênicos devem-se a anormalidades na migração do tecido ectomensequimal, no fluxo sangüíneo intracardíaco, na matriz celular, no crescimento alvo, no situs e looping cardíaco e na morte celular (ROSE; CLARCK, 1992). 
Aproximadamente $40 \%$ dos neonatos com cardiopatia congênita são portadores de malformações cardíacas complexas, isto é, apresentam associações de defeitos graves intracavitários e/ou dos grandes vasos. Estas formas de cardiopatia são a causa principal de mortalidade no período neonatal e na infância, representando aproximadamente $50 \%$ do total das causas de óbito por malformações congênitas (HOFFMAN; CHRISTIANSON, 1978; GEMBRUCH, 1997).

Embora a evolução da cardiologia pediátrica possibilite o diagnóstico e tratamento das cardiopatias congênitas após o nascimento, a elevada morbimortalidade no período perinatal decorrente dessas anomalias, determinou a necessidade de uma metodologia de diagnóstico pré-natal que permitisse a programação de uma melhor assistência ao feto e ao neonato portador destas malformações.

Na década de 1980, a melhoria da qualidade dos equipamentos de ultrasonografia permitiu a aquisição de imagens bidimensionais em tempo real e surgiram os primeiros relatos da correlação da anatomia cardíaca fetal normal com as imagens adquiridas em tempo real pelo ecocardiograma fetal (ALLAN et al., 1980; SAHN et al., 1980).

O ecocardiograma permitiu a avaliação do anatomofuncional do coração fetal, a partir do segundo trimestre da gestação, constituindo-se no principal método de diagnóstico das malformações cardíacas fetais com excelente correlação com o diagnóstico pós-natal. Este método possibilitou a programação de melhor assistência tanto no período pré-natal como no período pós-natal, com o objetivo de melhorar o prognóstico das cardiopatias congênitas (ALLAN et al., 1984; ALLAN et al., 1994). 
O ecocardiograma fetal era, em princípio, indicado quase que exclusivamente na presença de fatores de risco materno para ocorrência de cardiopatias ou na presença de outras malformações fetais diagnosticadas pelo ultra-som obstétrico. Porém, a elevada prevalência destas malformações observada em neonatos, na ausência destes fatores de risco, determinou a necessidade de um programa de detecção destes defeitos que pudesse abranger todas as gestantes.

Ainda na década de 1980, teve início na França, uma forma simples de rastreamento das malformações cardíacas que consistiu na avaliação do coração fetal durante a realização do ultra-som de rotina, mediante a utilização do corte ecocardiográfico quatro câmaras (FERMONT et al., 1986). A aplicação deste programa de rastreamento em diferentes centros mostrou-se eficaz na detecção das cardiopatias em $60 \%$ a $80 \%$ das gestantes avaliadas (COPEL et al., 1987; SILVERMAN; KLEINMAN CS, 1987). Entretanto, estudos posteriormente realizados, demonstraram que a aplicação de dois outros cortes ecocardiográficos, o plano eixo longo e eixo curto eram imprescindíveis para o diagnóstico de determinadas malformações e estes planos foram incorporados ao rastreamento ultra-sonográfico para possibilitar a detecção de cardiopatias com o comprometimento das grandes artérias. O acréscimo desta abordagem no rastreamento ultra-sonográfico das cardiopatias congênitas possibilitou alcançar taxas de detecção superiores a 80\% (ACHIRON et al; 1992; KIRK et al., 1994).

O rastreamento ultra-sonográfico das cardiopatias congênitas fetais constitui-se no principal meio de detecção desses defeitos no segundo semestre em gestantes de baixo risco. (DE VORE, 1985; COPEL et al., 1987, ALLAN, et al., 1994; ALLAN, 2000), Entretanto, Choui (2003) em uma revisão da literatura demonstrou que as 
taxas de detecção, em diferentes centros, variaram entre 5\% e 92\%. Estas variações devemse a diferenças na tecnologia disponível e, principalmente, do nível de treinamento dos ultra-sonografistas na obtenção e interpretação das imagens cardíacas. Isso demonstra a importância dos programas de capacitação destes profissionais para a realização de um rastreamento efetivo (CARVALHO et al., 2002, LOPES et al., 2003a).

A avaliação cardíaca fetal foi então dividida em dois níveis: no nível I, a avaliação cardíaca é realizada por ultra-sonografia obstétrica com o intuito de rastrear as malformações cardíacas fetais. No nível II, a avaliação cardíaca é realizada por um ecocardiografista fetal, com o objetivo de estudar a anatomia e fisiologia cardiovascular e estabelecer o diagnóstico diferencial das diferentes cardiopatias. Tem sido considerada como melhor fase para a avaliação cardíaca, em ambos os níveis, o segundo trimestre da gestação, devido a maior facilidade de obtenção dos diferentes planos ecocardiográficos.

Até o final da década de 1980, a avaliação ultra-sonográfica geral, no primeiro trimestre só era possível para a determinação da idade gestacional por mensuração do tamanho do feto, determinação de número de fetos (gestação única ou múltipla), confirmação de vida fetal e detecção de possíveis malformações grosseiras nesta fase, como a anencefalia.

Nos últimos anos, os avanços na tecnologia ultra-sonográfica, principalmente, na ultra-sonografia transvaginal, tem permitido obter um conhecimento mais preciso da embriologia fetal, identificar malformações congênitas nos primeiros meses de gestação e verificar a presença dos marcadores ultra-sonográficos de anomalias 
cromossômicas, colaborando para uma maior compreensão da fisiopatologia das diversas condições fetais.

Assim, Nicolaides et al. (1992) descreveram a relação entre o aumento do tamanho da transluscência nucal (coleção de líquido linfático localizada na região nucal do feto) e a presença de aneuploidias, principalmente as trissomias 13, 18 e 21. Esta alteração constitui um marcador de anomalias fetais e pode ser vizibilizada ao exame de ultra-som, entre a $11^{\mathrm{a}}$ e a $13^{\mathrm{a}}$ semana completa de gestação, época em que o comprimento cabeçanádega situa-se entre 45 e 84 milímetros.

Alguns trabalhos demonstraram, posteriormente, a associação entre o aumento da translucência nucal e a presença de cardiopatia estrutural e/ou alteração dos diâmetros dos grandes vasos com redução do istmo aórtico em fetos com aneuploidias e, também, em fetos euplóides (HYETT et al., 1995a; HYETT et al., 1995b; HYETT et al., 1996; SOUKA; NICOLAIDES, 1997; PANDIA et al., 1995).

Uma possível explicação para a associação entre o aumento da translucência nucal e as anormalidades do coração e dos grandes vasos é a presença de redução da complacência cardíaca nesta fase com conseqüente insuficiência diastólica (HYETT et al., 1997).

A utilização da medida da translucência nucal para o rastreamento das cardiopatias congênitas foi enfatizada por Hyett et al. (1999), em um estudo de coorte retrospectivo que analisou 29.153 fetos euplóides. Esses autores utilizaram como valor de corte o percentil 95 da medida da translucência nucal, considerando uma espessura de 2,2 
mm para fetos com comprimento crânio-nádegas de 38 milímetros (mm) e de 2,8 mm para um comprimento crânio-nádegas de 84 mm.

Os valores preditivos positivo e negativo para detecção de cardiopatias na presença de medida da translucência maior que o percentil 95 foram de 1,5 e 99,9 respectivamente, com sensibilidade de 56\% e especificidade de 94\%. Quando foi utilizado o percentil 99, como valor de corte, houve um incremento da especifidade de 99\%, porém a sensibilidade dimimuiu a 40\%, com valor preditivo negativo de $99 \%$.

A prevalência total de cardiopatias na presença de translucência nucal aumentada foi de 20/1.000, sendo importante destacar que houve correlação entre a medida da translucência e a prevalência de cardiopatias. Para valores maiores que 3,4 mm, a prevalência foi de 5,3/1.000 e, para medidas superiores a 5,5 mm, a prevalência foi de 195/1.000.

Outra importante contribuição da abordagem ultra-sonográfica do primeiro trimestre foi a avaliação Doppler do fluxo do ducto venoso que representa a medida da pressão venosa central fetal. A presença de alterações deste fluxo, como fluxo reverso ou ausente, durante a contração atrial, foi observada em fetos com translucência nucal aumentada (MATIAS et al., 1999) e em fetos euplóides com malformações estruturais, principalmente, cardíacas (BILARDO et al., 2000; ANTOLIN et al., 2001).

A detecção dessas alterações ultra-sonográficas, no primeiro trimestre, foi importante para o desenvolvimento de um programa de rastreamento de aneuploidias e malformações pela Fetal Medicine Foundation que atualmente é utilizado em vários centros de medicina fetal (SMALL; COPEL, 2004). 
A realização do ecocardiograma fetal no primeiro trimestre, na presença dos marcadores ultra-sonográficos e outros fatores de alto risco para anomalias cardíacas (idade materna acima de 35 anos, história familiar de malformações gênicas ou cromossômicas), tem demonstrado elevada prevalência de cardiopatias congênitas fetais neste grupo (GEMBRUCH et al., 1990; JOHNSON et al., 1992; ACHIRON et al., 1994a; HAAK et al., 2002; GABRIEL et al., 2002; SIMPSON et al., 2000; LOPES et al., 2003; MAKRYDINAS et al., 2005).

A observação de alterações na simetria das câmaras cardíacas, das grandes artérias, bem como a presença de insuficiência da valva tricúspide, tem sido relacionada à presença de anomalias cromossômicas e cardiopatias graves no primeiro trimestre da gestação (HYETT et al., 1995; HUGGON et al., 2001; DE VORE, 2002). Entretanto, poucos estudos sobre as características normais do coração fetal nesta fase foram realizados (ACHIRON et al., 1994b; ALLAN et al., 1997; ACHIRON et al., 1998; GEMBRUCH et al., 2000; HAAK et al., 2002, SMRCEK et al, 2006), não havendo parâmetros consensuais para a obtenção dos diferentes cortes ecocardiográficos, do período ideal para a avaliação cardíaca e padrões de normalidade das dimensões cardíacas, justificando o estudo de séries maiores com o objetivo de estabelecer esses critérios.

Por outro lado, a elevada prevalência de malformações cardíacas fetais, também observada em gestantes de baixo risco pode justificar a necessidade de um rastreamento ecocardiográfico no primeiro trimestre, porém não há ainda consenso, entre os especialistas em medicina fetal, quanto aos critérios de indicação nesta fase. 
Avanços no estudo da fisiopatologia das cardiopatias fetais e na sua detecção e diagnóstico precoce têm incrementado o tratamento de diversas doenças cardíacas congênitas, durante o período intra-útero. Recentemente, a valvoplastia aórtica por cateterismo, realizada no final do primeiro e início do segundo trimestre, demonstrou uma redução na taxa de progressão desta doença a sua forma mais grave, a síndrome de hipoplasia do coração esquerdo (LOPES et al, 1996; TWORETZKY et al., 2004).

Dessa forma, os avanços que permitam um maior conhecimento da anatomia e fisiologia cardiovascular fetal, no primeiro trimestre, podem ser determinantes para o aconselhamento genético. Além disso, serão fundamentais na seleção dos pacientes para tratamento intra-útero (medicamentoso ou intervencionista), com o objetivo de reduzir a mortalidade fetal e melhorar o prognóstico pós-natal. 


\section{OBJETIVOS}




\section{OJETIVOS}

Os objetivos deste trabalho foram:

1. Determinar o índice de sucesso na realização do estudo anatômico completo do coração fetal entre $11^{\mathrm{a}}$ e a $14^{\mathrm{a}}$ semanas, por via transvaginal, em fetos com baixo risco para cardiopatias.

2. Avaliar qual a melhor fase deste período para a obtenção dos principais cortes ecocardiográficos.

3. Estudar os aspectos anatômicos e determinar os diâmetros dos anéis valvares atrioventriculares e ventriculoarteriais do coração fetal.

4. Comparar os diâmetros obtidos em cada uma das quatro semanas com o comprimento crânio-nádegas. 


\section{POPULAÇÃO E MÉTODOS}




\section{POPULAÇÃO E MÉTODOS}

\section{1.- População}

No período de julho de 2004 a julho de 2005, 237 gestantes foram encaminhadas da Rede Municipal de Saúde ao Ambulatório de Morfologia Fetal do Departamento de Ginecologia e Obstetrícia do Hospital das Clínicas da Faculdade de Medicina de Ribeirão Preto para inclusão no programa de rastreamento ultra-sonográfico de malformações, no primeiro trimestre, estabelecido pela Fetal Medicine Foundation. Desta população, 54 gestantes foram avaliadas por ecocardiografia transvaginal seriada, por um período de quatro semanas, num estudo prospectivo aprovado pelo Comitê de Ética do Hospital. Das 54 gestantes selecionadas, oito (15\%) abandonaram o seguimento, sendo excluídas do estudo. Nossa amostra foi constituída de 46 gestantes com idade de 16 a 34 anos (27,3 média \pm 6,6 desvio-padrão) de acordo com os critérios de inclusão previamente estabelecidos.

\section{1- Critérios de inclusão}

1. Pacientes com 11 semanas de gestação, segundo a data da última menstruação, (DUM) e medida do comprimento crânio-nádega do feto (CCN).

2. Ausência de fatores de risco materno para cardiopatia.

3. Exame ultra-sonográfico prévio sem evidências de malformações fetais.

4. Translucência nucal normal.

5. Dopplerfluxometria do ducto venoso normal 


\section{2- Critérios de exclusão}

1. Presença de qualquer alteração cardíaca detectada pelo ecocardiograma fetal

2. Abandono do seguimento sem completar o período estabelecido no protocolo

Após receberem informações detalhadas sobre o estudo, um Termo de

Consentimento Livre e Esclarecido previamente aprovado pela Comissão de Ética em Pesquisa da instituição (Processo No 361/2004) foi apresentado às pacientes.

Foi realizado um projeto-piloto em pacientes com 10 semanas de idade gestacional e devido à baixa resolução das imagens e à dificuldade na obtenção dos cortes ecocardiográficos, estes casos foram excluídos do presente estudo.

Dados referentes à identificação da paciente e informações sobre os antecedentes pessoais foram anotados em uma ficha protocolar individual, e os principais dados foram armazenados num banco de dados.

\section{2 - Métodos}

\section{1 - Equipamento}

Os exames foram realizados com um equipamento de alta resolução HDI 3000, Advanced Technology Laboratories, Bothell, WA, USA. Foram usados transdutores multifreqüênciais, sendo um convexo de 2-4 megahertz para realização do estudo ultrasonográfico obstétrico por via transabdominal e um transdutor de 4-8 megahertz para a obtenção do exame obstétrico e do ecocardiograma transvaginal. 


\section{2 - Avaliação ultra-sonográfica}

A avaliação ultra-sonográfica do primeiro trimestre da gestação foi realizada por meio de dois estudos, um estudo ultra-sonográfico obstétrico e um estudo ecocardiográfico fetal.

O estudo ultra-sonográfico obstétrico por via transvaginal foi realizado para a determinação da idade gestacional pelo comprimento crânio-nádegas, mensuração da translucência nucal e realização do estudo do fluxo do ducto venoso por Dopplerfluxometria.

Todas as 46 gestantes foram submetidas a quatro estudos ecocardiográficos fetais seqüenciais com intervalos de uma semana, sendo o primeiro imediatamente a seguir da realização do estudo ultra-sonográfico obstétrico.

Para a avaliação ecocardiográfica fetal, utilizou-se exclusivamente a via transvaginal e os exames foram realizados por um único profissional (SRMC). A duração do exame foi limitada em 20 minutos para segurança do feto e conforto da paciente.

O estudo ecocardiográfico completo foi realizado utilizando-se o Modo Bidimensional para a obtenção dos seguintes cortes:

1. Quatro câmaras: este corte permite o estudo das conexões venoatriais e atrioventriculares, da junção átrioventricular, dos septos intracavitários e das cavidades ventriculares. A mensuração dos anéis átrioventriculares foi realizada durante a diástole ventricular, incluindo-se a parede da junção atrioventricular e excluindo-se o septo interatrial, sendo os valores expressos em milímetros (figura 1A). 
2. Eixo longitudinal: este corte possibilita a determinação da conexão ventriculoarterial posterior representada pela valva aórtica, e a continuidade anatômica deste vaso com o septo interventricular. A mensuração do diâmetro do anel valvar aórtico foi realizada durante a sístole ventricular, incluindo-se as paredes do vaso, sendo a medida expressa em milímetros (figura 1B).

3. Eixo curto: este corte foi utilizado para a visibilização da conexão ventriculoarterial anterior que corresponde à valva pulmonar. A mensuração do diâmetro do anel valvar pulmonar foi realizada durante a sístole ventricular, incluindo-se a parede externa do vaso e excluindo-se a parede interna, sendo os valores expressos em milímetros (figura 1C).
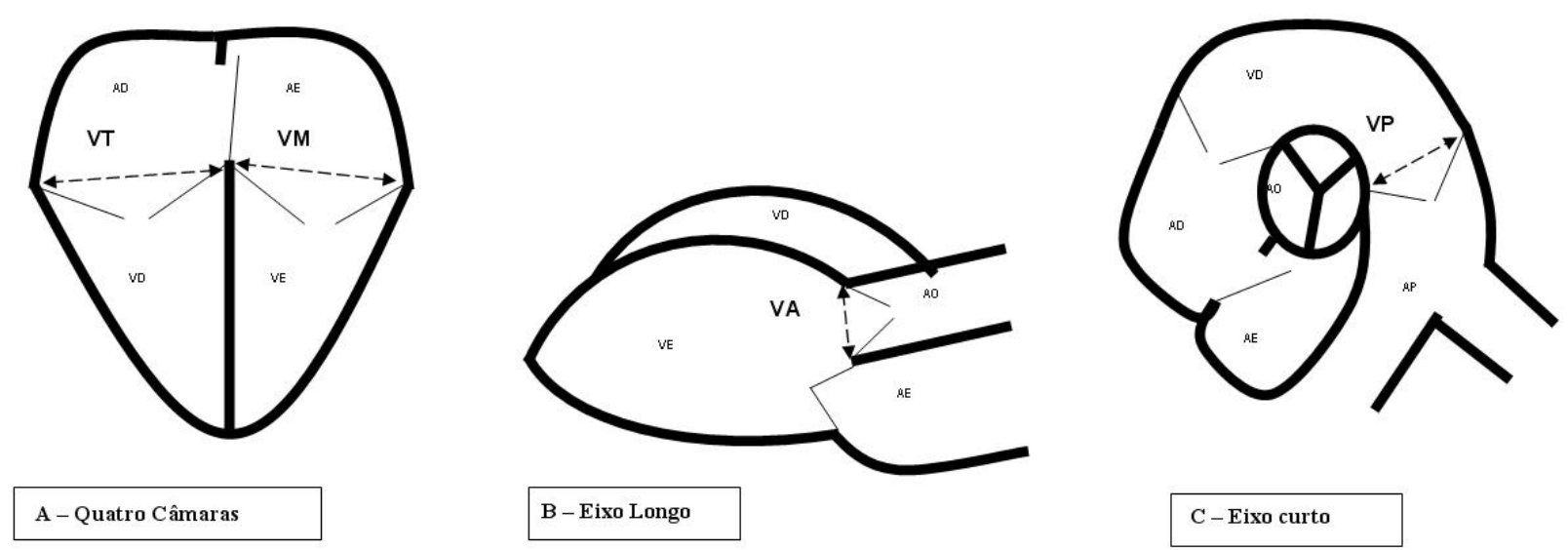

Figura 1: Desenho esquemático dos cortes ecocardiográficos básicos utilizados no estudo demonstrando como foram realizadas as medidas dos diâmetros. 1-A: Plano quatro câmaras: utilizado para determinação dos diâmetros das valvas atrioventriculares 1-B: Eixo longo: para mensuração do diâmetro do anel valvar aórtico. 1-C: Eixo curto: para a mensuração do diâmetro do anel valvar pulmonar.

VAO: valva aórtica, VD: ventrículo direito, VE: ventrículo esquerdo, VM: valva mitral, VT: valva tricúspide, VP: valva pulmonar. 
As medidas dos diâmetros valvares e do comprimento crânio-nádegas foram obtidas da média de três determinações: uma medida durante a realização do exame e outras duas medidas por revisão das imagens gravadas.

A avaliação cardiovascular foi complementada com a determinação do situs visceral mediante a relação espacial dos órgãos abdominais, da aorta abdominal, das veias cavas e do ducto venoso e da posição cardíaca, segundo a orientação do apex cardíaco na cavidade torácica.

Um novo estudo ecocardiográfico fetal por via transabdominal foi realizado entre a $20^{\mathrm{a}}$ e a $23^{\mathrm{a}}$ semanas de idade gestacional, para confirmar a ausência de alterações cardíacas.

Dados relacionados ao parto e condição clínica dos recém-nascidos foram obtidos por análise dos prontuários ou informações fornecidas pelas mães.

\section{3 - Processamento digital da imagem e banco de dados}

Os dados de identificação das pacientes e as imagens ecocardiográficas capturadas (Figura 2) e gravadas em sistema DICOM (Digital Imaging and Communications) foram arquivados em um banco de imagens pelo programa WorkstationMerge-efilm ${ }^{T M}$ 1.8.3-1998-2002, USA, o que permitiu uma rápida localização e revisão. 

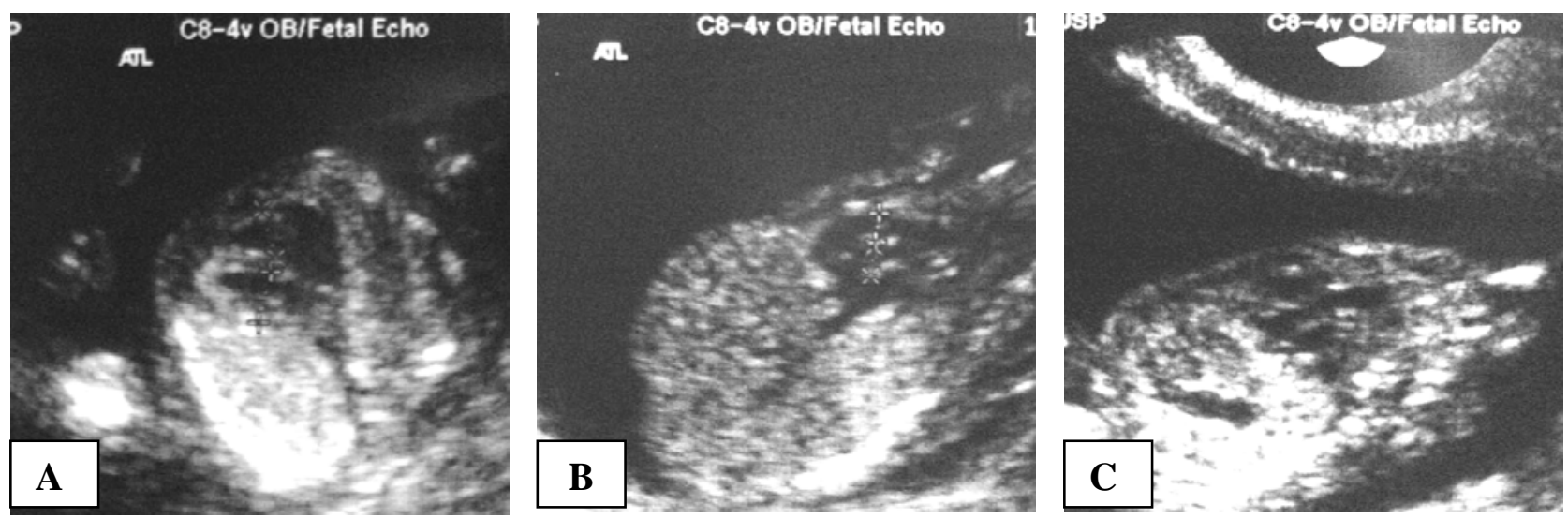

Figura 2: Imagens obtidas dos cortes ecocardiográficos básicos utilizados: A: Plano quatro câmaras, B: Eixo longo, C: Eixo curto.

\section{4 - Análise estatística}

As curvas de crescimento foram construídas mediante o software GraphPadPrism ${ }^{\circledR}$, versão 3.00, USA, utilizando-se os diâmetros, expressos em milímetros, dos anéis valvares mitral, tricúspide, aórtico e pulmonar e do CCN, obtidos nos exames semanais de cada feto. A idade gestacional foi calculada em semanas e dias, de acordo com a medida do CCN.

A equação de regressão dos diâmetros dos anéis valvares foi modulada em função do CCN. O teste de Pearson foi realizado para medir a força de associação entre as duas variáveis (diâmetros dos anéis valvares e CCN) e foi expressa como coeficiente de correlação $\left(\mathrm{r}^{2}\right)$. O nível de significância utilizado foi de 5\%.

Foram determinadas, semanalmente, a taxa de sucesso na obtenção do estudo ecocardiográfico e a porcentagem de obtenção dos diferentes cortes ecocardiográficos.

As variáveis contínuas foram descritas por média \pm desvio-padrão e limites superior e inferior. 
RESULTADOS 


\section{RESULTADOS}

A idade materna variou entre 16 e 34 anos $(M=27 \pm 6,6$ anos), e a paridade variou de zero a quatro (mediana 2). Foram realizados 184 estudos ecocardiográficos, sendo quatro exames para cada uma das 46 participantes com intervalos semanais.

A partir da $11^{\text {a }}$ semana de gravidez, foi possível em todos os casos a determinação do situs visceral e da posição cardíaca fetal, evidenciando-se em todos os casos, situs solitus, levoapex e conexões interseguimentárias concordantes do coração e grandes vasos. O ecocardiograma fetal transabdominal realizado entre a $20^{\mathrm{a}}$ e a $25^{\mathrm{a}}$ semanas confirmou a normalidade anatomofuncional e as características anatômicas observadas no primeiro trimestre, em $100 \%$ dos casos.

A percentagem de sucesso, na realização de um estudo ecocardiograma fetal completo com a obtenção dos três cortes principais, foi de $37 \%$ na $11^{\text {a }}$ semana, $85 \%$ na $12^{\text {a }}$ semana, e de $100 \%$ entre a $13^{\mathrm{a}}$ e a $14^{\mathrm{a}}$ semanas. Nas duas primeiras semanas, o corte mais facilmente obtido foi o eixo longo e o de mais difícil visibilização, o eixo curto (Tabela 1).

Tabela 1: Taxas percentuais de realização dos principais cortes ecocardiográficos, de acordo com a idade gestacional em semanas (CCN) e taxas de sucesso na realização de todos os cortes (completo).

\begin{tabular}{|c|c|c|c|c|c|c|c|c|}
\hline \multirow[t]{2}{*}{ IG (semanas) } & \multicolumn{2}{|c|}{ Quatro câmaras } & \multicolumn{2}{|c|}{ Eixo curto } & \multicolumn{2}{|c|}{ Eixo longo } & \multicolumn{2}{|c|}{$\begin{array}{c}\text { Exame } \\
\text { completo }\end{array}$} \\
\hline & $\mathrm{N}$ & $\%$ & $\mathrm{~N}$ & $\%$ & $\mathrm{~N}$ & $\%$ & $\mathrm{~N}$ & $\%$ \\
\hline 11 & 31 & 67 & 20 & 43 & 40 & 87 & 17 & 37 \\
\hline 12 & 43 & 93 & 41 & 89 & 46 & 100 & 39 & 85 \\
\hline 13 & 46 & 100 & 46 & 100 & 46 & 100 & 46 & 100 \\
\hline 14 & 46 & 100 & 46 & 100 & 46 & 100 & 46 & 100 \\
\hline
\end{tabular}

IG: idade gestacional.

O CCN variou de $40 \mathrm{~mm}$ na $11^{\mathrm{a}}$ semana, a $91 \mathrm{~mm}$ na $14^{\text {a }}$ semana, demonstrando o rápido crescimento neste período em que duplica seu tamanho. A média do CCN nas quatro 
semanas foi de 47, 59, 70 e $82 \mathrm{~mm}$, respectivamente (Tabela 2). Foi observada uma taxa de sucesso de 100\% na realização de um estudo ecocardiográfico completo, quando o CCN foi igual ou superior a 65 mm ou 13 semanas de gestação (Tabela 2).

Tabela 2: Descrição das variáveis relacionadas com o ecocardiograma fetal nas semanas $11^{\mathrm{a}}, 12^{\mathrm{a}}, 13^{\mathrm{a}}$ e $14^{\mathrm{a}}$ semanas de gestação.

\begin{tabular}{ccccccccc}
\hline Variável & Tempo & N & Média & DP & CV & \multicolumn{3}{c}{ Mínimo } \\
\hline CCN & 11 & 46 & 47,11 & 3,14 & 6,66 & 40,60 & 47,20 & 52,80 \\
& 12 & 46 & 58,85 & 4,82 & 8,18 & 46,20 & 59,10 & 67,70 \\
& 13 & 46 & 70,26 & 3,58 & 5,12 & 65,00 & 69,15 & 76,70 \\
& 14 & 46 & 82,45 & 3,83 & 4,65 & 76,10 & 81,45 & 91,00 \\
AVM & 11 & 21 & 2,72 & 0,26 & 9,64 & 2,10 & 2,70 & 3,20 \\
& 12 & 42 & 3,20 & 0,29 & 9,06 & 2,60 & 3,30 & 3,70 \\
& 13 & 46 & 3,71 & 0,31 & 8,38 & 2,90 & 3,80 & 4,40 \\
& 14 & 46 & 4,26 & 0,42 & 9,83 & 3,40 & 4,20 & 5,20 \\
AVT & 11 & 21 & 2,76 & 0,29 & 10,50 & 2,20 & 2,70 & 3,40 \\
& 12 & 42 & 3,25 & 0,29 & 8,85 & 2,60 & 3,30 & 3,80 \\
& 13 & 46 & 3,70 & 0,31 & 8,50 & 3,00 & 3,70 & 4,50 \\
& 14 & 46 & 4,31 & 0,42 & 9,74 & 3,50 & 4,20 & 5,30 \\
AVAo & 11 & 29 & 1,53 & 0,17 & 11,16 & 1,20 & 1,50 & 2,10 \\
& 12 & 46 & 1,86 & 0,20 & 10,51 & 1,50 & 1,90 & 2,30 \\
& 13 & 46 & 2,19 & 0,17 & 7,71 & 1,80 & 2,20 & 2,50 \\
& 14 & 46 & 2,50 & 0,14 & 5,81 & 2,20 & 2,50 & 2,90 \\
AVP & 11 & 20 & 1,55 & 0,17 & 11,27 & 1,20 & 1,60 & 2,00 \\
& 12 & 31 & 1,87 & 0,18 & 9,85 & 1,50 & 1,90 & 2,30 \\
& 13 & 46 & 2,20 & 0,18 & 8,20 & 1,90 & 2,20 & 2,60 \\
& 14 & 46 & 2,52 & 0,16 & 6,27 & 2,20 & 2,50 & 3,00 \\
\hline
\end{tabular}

AVM: Anel valvar mitral, AVT: anel valvar tricúspide, CCN: comprimento crânio-nádegas, AVAO: anel valvar aórtico, AVP: anel valvar pulmonar, N: tamanho da amostra, DP: desvio-padrão, CV: coeficiente de variação.

Dos 184 exames realizados, a determinação dos diâmetros dos anéis valvares mitral, tricúspideo, aórtico e pulmonar foi possível em 155 (84\%), 155 (84\%), 167 (89\%) e 143 (77\%), respectivamente, demonstrando que o diâmetro do anel valvar pulmonar foi o mais difícil de ser visibilizado. O diâmetro do anel valvar aórtico foi o mais facilmente visibilizado, permitindo a obtenção das medidas, em todos os casos, a partir da $12^{\mathrm{a}}$ semana. 
Não houve diferença estatisticamente significativa na comparação entre a média dos diâmetros dos anéis valvares mitral e tricúspide $(p<0,001)$, indicando tamanho e crescimento semelhante de ambos, nesta fase do desenvolvimento fetal. O mesmo resultado foi obtido, quando foram comparados os diâmetros dos anéis valvares aórtico e pulmonar (Tabela 2).

Foram realizadas correlações entre os diâmetros valvares atrioventriculares e a idade gestacional sendo observado que houve correlação positiva entre os valores correlacionados. Os valores do coeficiente de correlação $\left(\mathrm{r}^{2}\right)$ foram de 0,74 e 0,75 para os diâmetros valvares mitral e tricúspide, respectivamente (Tabela 3). Isto indica uma forte correlação entre estes diâmetros e idade gestacional, com significância estatística ( $p$ $<0,001)$. Comportamento semelhante foi observado entre as variáveis dos diâmetros valvares aórtico e pulmonar e o CCN, havendo uma correlação positiva. Os coeficientes de correlação $\left(\mathrm{r}^{2}\right)$ foram de 0,77 para o diâmetro valvar aórtico e de 0,73 para o diâmetro valvar pulmonar (Tabela 3). Isto indica uma forte correlação entre estes diâmetros e idade gestacional com significância estatística (p <0,001).

Tabela 3: Equação de regressão linear, valor de r, valor de $p$ e n para cada variável.

\begin{tabular}{lcccc}
\hline Variável & Equação Linear & $\mathrm{r}^{2}$ & $\mathrm{p}$ & $\mathrm{n}$ \\
\hline AVM & $\mathrm{y}=-0,8218+0,0411 \mathrm{x}$ & 0,7414 & $<0,001$ & 155 \\
AVT & $\mathrm{y}=-0,8308+0,0415 \mathrm{x}$ & 0,7502 & $<0,001$ & 155 \\
AVAO & $\mathrm{y}=-0,3697+0,0255 \mathrm{x}$ & 0,7719 & $<0,001$ & 167 \\
AVP & $\mathrm{y}=-0,4002+0,0253 \mathrm{x}$ & 0,7312 & $<0,001$ & 143 \\
\hline
\end{tabular}

AVM: Anel valvar mitral, AVT: anel valvar tricúspide, AVAO: anel valvar aórtico, AVP: anel valvar pulmonar, r²: coeficiente de correlação, p: nível de significância, n: tamanho da amostra. 
Foram construídas curvas de crescimento, utilizando-se as variáveis dos diâmetros dos anéis valvares mitral e tricúspide (Figura 3) e aórtico e pulmonar (Figura 4) e o CCN, sendo observada uma curva de crescimento linear.
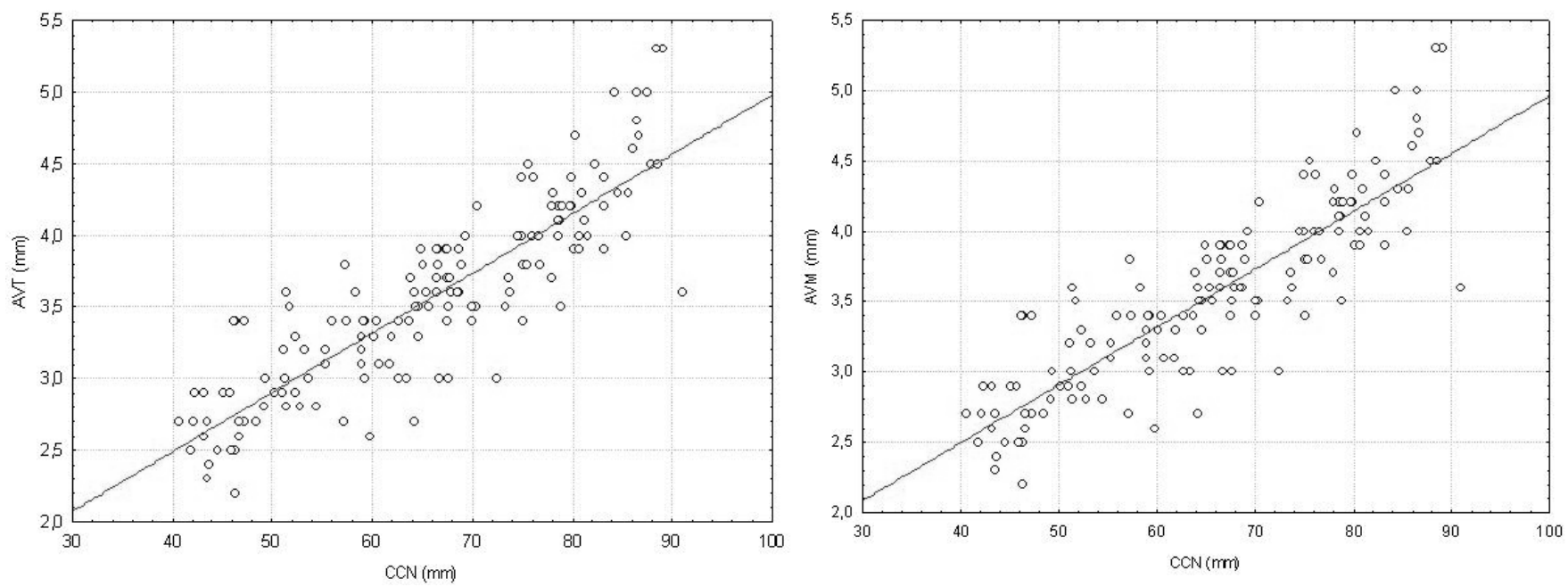

Figura 3: Relação entre os diâmetros dos anéis valvares mitral, tricúspide e o comprimento crânio-nádegas em milímetros.

AVM: Anel valvar mitral, AVT: anel valvar tricúspide, CCN: comprimento crânio-nádegas.
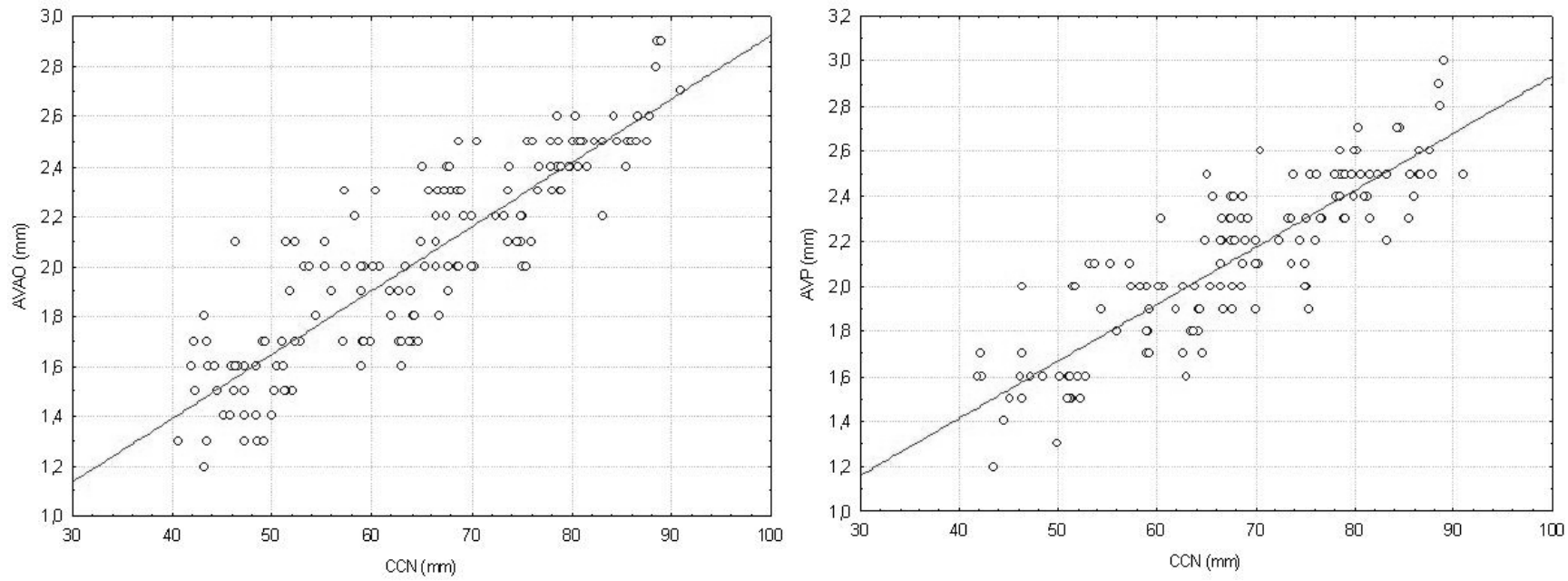

Figura 4: Relação entre os diâmetros dos anéis valvares aórtico e pulmonar e o comprimento crânio-nádegas em milímetros.

AVAO: anel valvar aórtico, AVP: anel valvar pulmonar, CCN: comprimento crânio-nádegas. 
As avaliações clínicas pós-natais realizadas nos recém-nascidos não demonstraram alterações cardiovasculares. Um feto evoluiu com arritmia cardíaca periparto e a avaliação ecocardiográfica demonstrou presença de extrassistolia supraventricular esporádica sem repercussão clínica. Um recém-nascido apresentou sopro cardíaco na primeira semana de vida. Em ambos os casos, foram realizados estudos ecocardiográficos transtorácicos no período pós-natal que não evidenciaram alterações cardiovasculares. 


\section{DISCUSSÃO}




\section{DISCUSSÃO}

A ecocardiografia fetal realizada no primeiro trimestre da gestação ainda não apresenta padrões de normalidade e indicações bem definidas. Ao contrário, o exame realizado a partir do segundo trimestre constitui o principal método de diagnóstico das cardiopatias congênitas, com critérios bem definidos.

A detecção dos marcadores ultra-sonográficos de aneuploidias e malformações estruturais tem sido a principal indicação para realização da ecocardiografia fetal no primeiro trimestre, constituindo um complemento da avaliação fetal nesta fase (CARVALHO, 2004).

O coração fetal pode ser examinado a partir da nona semana por via transvaginal (ALLAN et al., 1997), entretanto, parece haver unanimidade entre os autores, em afirmar que melhores resultados podem ser obtidos a partir da $12^{\mathrm{a}}$ semana (DOLKART; REIMERS, 1991; GEMBRUCH; SHI; SMRCEK, 2000; HAAK et al., 2002; SMARCEK et al., 2006).

A mensuração ultra-sonográfica do CCN corresponde ao tamanho do feto no seu maior eixo e permite estimar a idade gestacional. Este dado pode ser considerado determinante para o sucesso na obtenção de um estudo ecocardiográfico completo e foi demonstrado em dois estudos, realizados por via transvaginal (GEMBRUCH et al., 2000, HAAK et al., 2002). Os melhores resultados foram obtidos quando o CCN foi superior a 60 mm, o que corresponde ao período compreendido entre o final da $12^{\mathrm{a}}$ semana e o início da $13^{\mathrm{a}}$ semana de gestação. Huggon et al. (2002), em um estudo realizado por via 
transabdominal, no primeiro trimestre, também obtiveram uma taxa de sucesso de $90 \%$, na avaliação do coração fetal, quando o CCN foi superior a 61,4 milímetros.

No presente estudo, obteve-se sucesso na realização de um ecocardiograma completo de $85 \%$ na $12^{\mathrm{a}}$ semana de gestação, quando a média do comprimento do CCN foi de $58 \mathrm{~mm}$. Obtuvimos taxa de sucesso de 100\% quando o comprimento foi superior a 65 mm, portanto compatível com a idade gestacional de 13 semanas. Isto demonstra que o tamanho do feto correlaciona-se com a obtenção de melhores resultados a partir deste padrão de medida.

O estudo ecocardiográfico fetal, no primeiro trimestre, pode ser realizado utilizando-se a via transabdominal (CARVALHO et al., 1998; HUGGON et al., 2002) ou a via transvaginal (GEMBRUCH et al., 1990; BRONSTEIN el al., 1991; JOHNSON, et al., 1992). Em geral, a preferência na utilização da via transvaginal em relação à via transabdominal deve-se à melhor definição de imagem proporcionada pela redução da distância entre o transdutor e o coração fetal e pela elevada freqüência sonora emitida por estes transdutores o qual proporciona melhor resolução espacial (HAAK, et al., 2002; BHAT; SAHN, 2004).

A realização do ecocardiograma fetal por via transvaginal pode representar uma limitação para o ecocardiografista, devido ao envolvimento de uma metodologia ginecobstétrica, pouco familiar ao cardiologista, exigindo deste uma curva de aprendizado (ALLAN, 2000; DE VORE, 2002).

No nosso estudo utilizou-se a via transvaginal, havendo um treinamento técnico prévio do ecocardiografista junto ao ultra-sonografista. Este treinamento consistiu 
no aprendizado da utilização da sonda transvaginal e das mensurações do comprimento crânio-nádegas e da translucência nucal e a avaliação Doppler do fluxo do ducto venoso.

Achiron et al. (1994b) obtiveram 100\% de sucesso na visibilização do plano quatro câmaras e 98\% de sucesso na obtenção dos demais cortes ecocardiográficos entre a $13^{\mathrm{a}}$ e a $15^{\mathrm{a}}$ semanas, utilizando a via transvaginal e sondas multifreqüenciais de 6,5 a 7,5 megahertz.

Mais recentemente, Haak et al. (2002) demonstraram uma taxa média de sucesso de 92\% na realização de um estudo ecocardiográfico completo por via transvaginal, utilizando transdutor multifreqüencial de 4 a 8 megahertz entre a $11^{\mathrm{a}}$ semana $(11+0)$ e a $13^{\mathrm{a}}$ semanas $(13+6)$. A taxa de obtenção do corte quatro câmaras aumentou de $85 \%$ na $11^{\text {a }}$ semana para $98 \%$ na $13^{\text {a }}$ semana. O eixo curto foi mais facilmente visibilizado que o eixo longo na $11^{\mathrm{a}}$ semana, porém esta diferença desapareceu na $13^{\mathrm{a}}$ semana, com uma taxa de visibilização de ambos os cortes de quase $100 \%$.

Na nossa série, utilizamos a via transvaginal com transdutores multifreqüenciais de 4 a 8 megahertz e obtivemos resultados semelhantes, com $100 \%$ de sucesso na realização de estudo ecocardiográfico completo a partir da $13^{\mathrm{a}}$ semana. Entre $11^{\mathrm{a}}$ e a $12^{\mathrm{a}}$ semanas, houve maior dificuldade na obtenção de todos os cortes ecocardiográficos, principalmente do plano quatro câmaras e eixo curto e o estudo completo foi possível em 50\% e 85\%, respectivamente. Esta dificuldade deve-se, possivelmente, à inter-relação de dois fatores, o pequeno tamanho do coração fetal nesta fase, dificultando a definição de estruturas com diâmetros inferiores ao limite de resolução axial do equipamento de ultrasonografia $(0,2 \mathrm{~mm})$ e a posição fetal em relação à orientação dos planos de corte ultra- 
sonográficos. Esta dificuldade, também foi relatada por Gembruch et al. (1990), em um dos primeiros trabalhos publicados com a utilização da via transvaginal para o estudo do coração fetal. Em outra série, Gembruch et al. (2000) compararam as duas vias, transvaginal e transabdominal, entre a $10^{\mathrm{a}}$ e $17^{\mathrm{a}}$ semanas, demonstrando que, na $13^{\mathrm{a}}$ e na $14^{\mathrm{a}}$ semanas, a via transabdominal apresentou uma taxa de sucesso de 80 a $90 \%$ respectivamente, porém a via transvaginal foi superior com uma taxa de sucesso de 100\% em ambos os períodos. Após a 14ª semana, as duas vias foram igualmente sensíveis.

O primeiro estudo para estabelecer padrões de normalidade das dimensões cardíacas no primeiro trimestre foi realizado por Allan et al. (1997) que compararam as imagens ecocardiográficas obtidas por via transvaginal, a partir da nona semana de gestação e as imagens de microdissecção anatômica do coração de embriões e fetos humanos, Estes autores demonstraram que, a partir da $11^{\text {a }}$ semana, os diâmetros da aorta e artéria pulmonar adquirem tamanhos aproximadamente iguais.

Achiron et al. (1998), em sua pesquisa para determinar o padrão de normalidade dos diâmetros da artéria pulmonar e da aorta entre a $14^{\mathrm{a}}$ e a $26^{\mathrm{a}}$ semanas, observaram que os diâmetros de ambos os vasos foram semelhantes, evidenciando uma curva de crescimento linear durante o período do estudo.

Gembruch et al. (2000) realizaram um estudo prospectivo para avaliar a biometria fetal, em 136 fetos, entre a $10^{\mathrm{a}}$ e a $17^{\mathrm{a}}$ semanas, observando que a relação entre os diâmetros dos ventrículos direito e esquerdo (VD/VE) e do tronco pulmonar e da aorta (TP/AO) foram 1,00 e 1,10 respectivamente, demonstrando a manutenção de um mesmo padrão de crescimento no primeiro e segundo trimestres. 
Haak et al. (2002), em uma série prospectiva, com uma população de 85 fetos avaliados por ecocardiografia transvaginal durante três semanas consecutivas e realizaram um total de 255 avaliações. Estes autores observaram que os diâmetros da aorta (medidos no plano valvar) e da artéria pulmonar (medidos na altura do plano supravalvar) apresentavam o mesmo padrão de crescimento linear entre a $11^{\mathrm{a}}(11+0)$ e a $13^{\mathrm{a}}$ semanas (13+6) da gestação. No entanto, o diâmetro da artéria pulmonar foi discretamente maior que o diâmetro da aorta em fetos sem anomalias cardíacas.

Em um estudo recente, Smrcek et al. (2006) analisaram, prospectivamente, a biometria cardíaca fetal entre a $10^{\mathrm{a}}$ e a $15^{\mathrm{a}}$ semanas em 123 fetos e observaram que o diâmetro da artéria pulmonar foi minimamente maior que o diâmetro da aorta, apresentando diâmetros médios de 1,99 mm e 1,75 mm respectivamente na $14^{\mathrm{a}}$ semana, havendo uma correlação linear entre estes diâmetros e a idade gestacional. Os diâmetros dos ventrículos direito e esquerdo também foram avaliados, apresentando o mesmo padrão de crescimento linear e uma relação constante no tamanho de ambos.

Na presente série, foram mensurados semanalmente os diâmetros dos anéis das valvas atrioventriculares e ventriculoarteriais, dos 46 fetos, durante o período entre a $11^{\mathrm{a}}$ e a $14^{\mathrm{a}}$ semanas de gestação e foi comparado o crescimento semanal destes diâmetros com o aumento do CCN. Como preconizado por Salomon et al. (2003), esta metodologia pode ser mais representativa, em termos de interpretação estatística, pois as variáveis biológicas foram avaliadas seqüencialmente em um mesmo indivíduo e por um período de tempo consecutivo. 
No nosso estudo, o diâmetro médio da valva pulmonar foi minimamente maior que o diâmetro médio da valva aórtica, porém esta diferença não teve significância estatística. Foi observado também um padrão de crescimento linear destes diâmetros em relação ao CCN. Estes resultados foram semelhantes aos obtidos por Haak et al (2002) e Smrcek et al (2006). Porém, os diâmetros médios dos anéis valvares aórtico e pulmonar obtidos no nosso trabalho foram maiores que os diâmetros referidos por esses autores. Esta diferença, possivelmente, deve-se aos diferentes pontos de referência utilizados para a realização da mensuração do coração fetal que, na nossa série, foi o plano valvar, enquanto os autores mencionados utilizaram o plano supravalvar, medindo o tamanho das grandes artérias.

A simetria entre as cavidades direitas e esquerdas e grandes artérias foi também observada em um estudo que avaliou 881 fetos, a partir da $17^{\mathrm{a}}$ semana de gestação (KIRK et al., 1999). Nos fetos sem cardiopatia, a relação entre os diâmetros do ventrículo direito e do ventrículo esquerdo (VD/VE) foi de 1,24, mantendo-se um padrão de crescimento linear constante com a evolução da gestação. De forma semelhante, a relação entre os diâmetros da artéria pulmonar e da aorta (AP/AO) foi de 1,41. As dimensões cardíacas direitas foram discretamente maiores que as esquerdas, mantendo-se esta relação e um padrão de crescimento linear constante até o final da gestação.

Na nossa série o diâmetro médio do anel valvar tricúspide foi minimamente maior que o da valva mitral, porém esta diferença não foi considerada estatisticamente significativa, havendo um padrão de crescimento linear constante durante o período de estudo. Não foi possível comparar este achado com outros dados da literatura, pois não há 
relatos da avaliação das dimensões das valvas atrioventriculares nesta fase do desenvolvimento fetal.

As determinações dos diâmetros das cavidades cardíacas não foram realizadas na nossa série devido a diversos motivos. Em primeiro lugar, a mensuração do diâmetro transverso das cavidades atriais encontra-se dificultada pela presença do forame oval que ocupa toda a porção central do septo interatrial, portanto não existe um ponto de referência para a realização de uma mensuração adequada. Em segundo lugar, a avaliação do tamanho das cavidades ventriculares pela determinação do diâmetro transverso pode não refletir a real dimensão destas cavidades, sobretudo na presença de estados patológicos, no qual existe uma alteração da geometria ventricular com alteração da relação eixo curto/eixo longo. Finalmente, a localização do ponto de referência no qual deverá ser realizada a medida do diâmetro transverso não está padronizada.

No presente estudo, não foi observada diferença qualitativa no tamanho das cavidades direitas e esquerdas, sendo concordante com a avaliação quantitativa dos diâmetros das valvas átrioventriculares mitral e tricúspide. Assim, a relação entre os diâmetros das valvas atrioventriculares, determinada no nosso estudo, pode ser representativa da relação existente entre o tamanho das cavidades direitas e esquerdas. Sendo o débito ventricular direito maior que o esquerdo na circulação fetal (RYCHIK, 2004), é válido inferir que, possivelmente uma avaliação quantitativa dos volumes das estruturas cardíacas, mediante o desenvolvimento de fórmulas geométricas, possa demonstrar alguma variação significativa destas câmaras durante esta fase. 
A correlação observada entre os diâmetros dos anéis valvares atrioventriculares e entre os diâmetros das valvas ventriculoarteriais, em fetos com anatomia cardíaca normal, representa um dado fundamental, podendo ser utilizada como valores de referência no rastreamento de cardiopatias neste período da gestação.

A maioria das malformações cardíacas é detectável no primeiro trimestre, entretanto algumas anomalias podem evoluir e tornarem-se mais aparentes no segundo ou terceiro trimestre como: as obstruções das vias de entrada ou saída dos ventrículos e arco aórtico, as hipoplasias dos ventrículos ou grandes artérias, os tumores, as disfunções primárias do miocárdio, as arritmias e a permeabilidade do forame oval ou ducto arterioso (TRINES; HORNBERGER, 2004). Esta característica evolutiva das cardiopatias pode representar uma limitação importante para a avaliação ecocardiográfica no primeiro trimestre, entretanto, a ausência de uma alteração nesta fase, não dispensa a realização do rastreamento ultra-sonográfico ou o estudo ecocardiográfico, no segundo ou terceiro trimestre, que deve ser obrigatória, fazendo parte da rotina pré-natal das gestantes. No presente estudo, todas as gestantes foram submetidas a uma nova avaliação ecocardiográfica no segundo trimestre, para confirmar a normalidade das estruturas cardíacas observadas no primeiro trimestre, confirmando a ausência de cardiopatia.

A realização do ecocardiograma fetal, no primeiro trimestre, pode aumentar a taxa de rastreamento das malformações cardíacas, principalmente nas gestantes de baixo risco, entretanto, este procedimento é ainda pouco freqüente, devido a importantes limitações como: falta de recursos tecnológicos e humanos especializados, carência de centros de treinamento e ausência de diretrizes das associações da especialidade para a implementação deste método de forma mais abrangente. 
A ampla utilização desse método pode permitir o diagnóstico precoce das malformações cardíacas, proporcionando o aconselhamento genético, o apoio psicológico aos familiares e a perspectiva de implantação de medidas terapêuticas que possam melhorar o prognóstico fetal e pós -natal.

No nosso meio, entretanto, a realização rotineira do ecocardiograma fetal no primeiro trimestre está por hora limitada a poucos centros de referência, e o benefício desta avaliação está restrito a poucos pacientes. 


\section{CONCLUSÕES}




\section{CONCLUSÕES}

Os resultados obtidos neste estudo nos permitem obter as seguintes conclusões:

1- O índice de sucesso na obtenção de um ecocardiograma fetal completo por via transvaginal foi baixo (37\%) na $11^{\mathrm{a}}$ semana, elevado na $12^{\mathrm{a}}$ semana $(85 \%)$ e de $100 \%$ na $13^{\mathrm{a}}$ e $14^{\mathrm{a}}$ semanas.

2- A medida do CCN igual ou superior a $65 \mathrm{~mm}$, compatível com a idade gestacional ultra-sonográfica de 13 semanas, foi determinante para obtenção de todos os cortes ecocardiográficos, demonstrando uma taxa de sucesso de $100 \%$, devendo ser esta a semana de escolha na realização dos estudos eletivos.

3- O estudo completo dos aspectos anatômicos do coração fetal foi possível a partir da $13^{\mathrm{a}}$ semana, quando foram obtidos todos os cortes ecocardiográficos na população estudada. Os diâmetros das valvas atrioventriculares e ventriculoarteriais foram determinados na $11^{\mathrm{a}}$ e $12^{\mathrm{a}}$ semana de acordo com a obtenção dos cortes ecocardiográficos e na totalidade da população na $13^{\mathrm{a}}$ e $14^{\mathrm{a}}$ semana.

4- Houve correlação positiva entre os diâmetros das valvas atrioventriculares e ventriculoarterias e o CCN com curvas de crescimento linear durante o período de avaliação. 


\section{REFERÊNCIAS BIBLIOGRÁFICAS}




\section{REFERÊNCIAS BIBLIOGRÁFICAS}

1. Achiron R, Glaser J, Gelenter I, Heggesh J, Yagel S. Extended fetal echocardiographic examination for detecting cardiac malformation in low-risk pregnancies. $\mathrm{Br}$ Med $\mathrm{J}$. 1992; 304:671-674.

2. Achiron R, Rotstein Z, Zipitz S, Mashiach S, Heggesh J. First trimester diagnosis of fetal congenital heart disease by transvaginal ultrasonography. Obstet Gynecol 1994; 84:69-72.

3. Achiron R, Weissman A, Rotstein Z, Lipitz S, Mashiach S, Hegesh J. Transvaginal echocardiographic examination of fetal heart between 13 to 15 weeks gestation in lowrisk population. J Ultrasound Med. 1994; 13:783-789.

4. Achiron R, Golan-Porat N, Gabbay U, Rotstein Z, Heggesh J, Lipitz S, Mashiach S. In uterus ultrasonographic measurements of fetal aortic and pulmonary artery diameters during the first half of gestation. Ultrasound Obstet Gynecol 1998; 11:180-184.

5. Allan LD, Tynan MJ, Campbell S, Wilkinson JL, Anderson RH. Echocardiographic and anatomical correlation in the fetal. Br Heart J 1980; 41:444-451.

6. Allan LD, Crawford DC, Anderson RH, Tynan MJ. Echocardiographic and anatomical correlation in fetal congenital heart disease. Br Heart J 1984; 52:542-548.

7. Allan LD, Sharland GK, Milbourn A, Lockharth SM, Groves AMM, Anderson RH, Cook AC. Prospective diagnosis of 1006 consecutive cases of congenital heart disease in the fetus. JAAC 1994; 23:1452-1458.

8. Allan LD, Santos R, Peixeder T. Anatomical and echocardiographic correlates of normal morphology of the late first trimester fetus. Heart 1997; 77:68-72. 
9. Allan LD. Congenital heart disease: antenatal diagnosis of heart disease. Heart 2000; 83: 367-370.

10. Antolin E, Comas C, Torrents M, Muñoz, A, Figueras F, Echevarría M, Cararach M, Carrera, J M M.The role of ductus venous flow assessment in screening for chromosomal abnormalities at 10-16 weeks of gestation. Ultrasound Obstet Gynecol $2001 ; 17: 295-300$.

11. Bhat A, Sahn J. Latest advances and topics in fetal echocardiography. Curr Opin Cardiol 2004; 19:97-103.

12. Bilardo CM, Müller MA, Zikulnig L, Schipper M, Hecher K. Ductus venous study in fetuses at high risk for chromosomal or heart abnormalities: relationship with nuchal translucency measurement and fetal outcome. Ultrasound Obstet Gynecol 2001; 17:288294.

13. Bronshtein M, Zimmer EZ, Milo S Ho SY, Lorber A, Gerlis LM. Fetal cardiac abnormalities detected by transvaginal sonography at 12-16 weeks gestation. Obstet Gynecol 1991; 78:374-378.

14. Carvalho JS, Moscoso G, Ville Y. First-trimestrer transabominal fetal echocardiography. Lancet. 1998; 351:1023-1027.

15. Carvalho JS, Mavrides E, Shinebourne EA, Campbell S, Thilaganathan B. Improving the effectiveness of routine prenatal screening for major congenital heart defects. Heart 2002; 88:387-391.

16. Carvalho JS. Fetal heart scanning in the first trimester. Prenat Diagn 2004; 24:10601067. 
17. Chooui R. Editorial: The four chamber view: four reasons why it seems to fail in screening for cardiac abnormalities and suggestions to improve detection rate. Ultrasound Obstet Gynecol 2003; 22:3-10.

18. Copel JA, Pilu G, Gren J, Hobbins JC, Kleiman CS. Fetal echocardiographic screening for congenital heart disease: the importance of the four-chamber view. Am J Obstet Gynecol.1987; 157:648-655.

19. DeVore GR. The prenatal diagnosis of congenital heart disease - a practical approach for the fetal sonographer. J Clin Ultrasound.1985; 13:229-245.

20. DeVore GR. First-trimester fetal echocardiography: is the future now? Opnion. Ultrasound Obstet Gynecol. 2002; 20:6-8.

21. Dolkart LA, Reimers FT. Transvaginal fetal echocardiography in early pregnancy: normative data. Am J Obstet Gynecol 1991; 165:688-691.

22. Fermont L, De Geeter B, Aubry J. A close colaboracion between obstetricians and pediatric cardiologists allows antenatal detection of severe cardiac malformation by 2D echocardiography. In: Doyle EF, Engle ME, Gersony WM (Eds). Pediactric cardiology: proceedings of the second world congress. New York: Springer Verlag 1986, p. 34.

23. Gabriel C, Galindo A, Martinez JM, Carrera JM, Gutierrez-Larraya F. Early prenatal diagnosis of major cardiac anomalies in a high risk population. Prenatal Diagn 2002; 22:586-593.

24. Gembruch U. Prenatal diagnosis of congenital heart disease. Prenat Diagn 1997; 17:1283-1298.

25. Gembruch U, Knopfle G, Chatterjee M, Bald R, Hansmann M. First trimester diagnosis of fetal congenital heart disease by transvaginal two-dimensional and Doppler echocardiography. Obstet Gynecol 1990; 75:496-498. 
26. Gembruch U, Shi C, Smarcek J. Biometry of fetal heart between 10 and 17 weeks gestation. Fetal Diag Ther 2000; 15:20-31.

27. Haak MC, Twisk JWR, Van Vugt JMG. How successful is fetal echocardiographic examination in first trimester of pregnancy. Ultrasound Obstet Gynecol 2002; 20:9-13.

28. Hoffman JL, Christianson R. Congenital heart disease in a cohort of 19.502 births with long-term follow-up. Am J Cardiol 1978; 42:641-647.

29. Hoffman JIE. Incidence of congenital heart disease: I. Postnatal incidence. Pediatr Cardiol 1995; 16:103-113.

30. Hoffman JIE. Incidence of congenital heart disease: II. Prenatal incidence. Pediatr Cardiol 1995; 16:155-165.

31. Hoffman JL, Kaplan S.The incidence of congenital heart disease. JAAC 2002; 39:18901900.

32. Huggon IC, Ghi T, Cook AC, Zosmer N, Allan LD, Nicolaides KH. Fetal cardiac abnormalities identified prior to 14 weeks gestation. Ultrasound Obstet Gynecol 2002; 20: 22-29.

33. Hyett JA, Moscoso G, Nicholaides KH. Morfhometric analyses of geat vessel in early fetal life. Hum Reprod 1995, 10:3045-3048.

34. Hyett JA, Moscoso G; Nicolaides KH. First trimester nuchal translucency and cardiac septal defects in fetuses with trisomy 21. Am. J. Obstet. Gynecol 1995, 172:1411-1413.

35. Hyett JA, Moscoso G; Nicolaides KH. Increased nuchal translucency in trisomy 21 fetuses: relationship to narrowing of the aortic isthmus. Human. Reprod. 1995; 10:30493051.

36. Hyett JA, Moscoso G, Papanagiotou G, Pardu M, Nicolaides KH. Abnormalities of the heart and great arteries in chromosomally normal fetuses with increased nuchal 
translucency thickness at 11-13 weeks of gestation. Ultrasound Obstet. Gynecol 1996, 7: 245-250.

37. Hyett JA, Perdu M, Sharland GK, Snijders RJM, Nicholaides KH. Increased of nuchal translucency at 10-14 weeks of gestational as a marker for major cardiac defects. Ultrasound Obstet Gynecol 1997; 10:242-246.

38. Hyett JA, Perdu M, Gurleen S, Snijders RJM, Nicholaides KH. Using fetal nuchal to screen major cardiac defects at 10-14 weeks of gestation population-based cohort study. Br Med J 1999; 318:81-85.

39. Johnson P, Sharland GK, Maxwell D, Allan LD. The role of transvaginal sonography in the early detection of congenital heart disease. Ultrasound Obstet Gynecol 1992; 2:248251.

40. Kirk JS, Comstock CH. Prenatal screening for cardaiac anomalies: the value of routine addition of aortic to the four-chamber view. Obstet Gynecol 1994; 84:427-431.

41. Kirk JS, Comstock CH, Lee W, Smith RS, Riggs TW, Weinhouse E. Fetal cardiac assymmetry: A marker for congenital heart disease. Obstet Gynecol 1999; 93:189-192.

42. Lopes LM, Cha SC, Kajita LJ, Aielo V, Zugaib M. Balloon dilatation of the aortic valve in the fetus: a case report. Fetal Diag Ther.1996; 11:296-300.

43. Lopes LM, Damiano AP, Zugaib M. Programa educativo de treinamento em ecocardiografia fetal nível I: impacto na referência e análise de resultados. Revista Brasileira de Ecocardiografia 2003; 3:61-67.

44. Lopes LM, Brizot ML, Lopes MAB, Ayelo VD, Schultz R, Zugaib M. Structural and functional cardiac abnormalities identified prior to 16 weeks gestation in fetuses with increased nuchal translucency. Ultrasound Obstet Gynecol 2003; 22:470-478. 
45. Makrydinas G, Sotiriadis A, Huggon IC, Simpson J, Ionnidis JP, Sharland G, Carvalho JS. Nuchal translucency and fetal cardiac defects: a pooled analysis of major fetal echocardiography centers. Am J Obstet Gynecol 2005; 192:89-95.

46. Matias A, Huggon J, Areias JC, Montenegro N, Nicolaides KH. Cardiac defects in chromosomally normal fetuses with abnormal ductus venosus blood flow at 10 to14 weeks. Ultrasound Obstet Gynecol 1999; 14:307-310.

47. Nicolaides KH, Snijders RJM, Gosten CM, Campbell S. Ultrasonographically detectatable markers of fetal chromosomal abnormalities. Lancet 1992; 340:704-707.

48. Pandia PP, Snijders RJM, Johnson SP, Brizot ML, Nicolaides KH. Screening for fetal trisomies by maternal age and fetal nuchal translucency thickness at 10-14 weeks of gestation. Br J Obstet Gynecol 1995; 102: 957-962.

49. Rose V, Clarck R. Etiology of congenital heart disease.In.Freedon RM, Leland BN, Smallhorn JIF (Eds). Neonatal heart disease. London: Springer Verlag 1992; p.3-17.

50. Rychik J. Fetal cardiovascular physiology. Pediatr Cardiol 2004; 25:201-209.

51. Sahn DJ, Lange LW, Allen HD. Quantitative real time cross-sectional echocardiography in the development of normal human fetus and newborn. Circulation 1980; 62:588-595.

52. Salomon LJ, Bernard JP, Duyme M, Ville Y. Revisiting first- trimester fetal biometry. Ultrasound Obstet Gynecol 2003; 22:63-66.

53. Silverman NH, Kleinman CS. Fetal echocardiographic screening for congenital heart disease: the importance of the four-chamber view. AM J Obstet Gynecol 1987, 157:648655. 
54. Simpson J, Jones A, Callaghan N, Sharland G. Accuracy and limitations of transabdominal fetal echocardiography at 12-15 weeks gestation in a population at high risk for congenital heart disease. Br J Obstet Gynaecol 2000; 107:1492-1497.

55. Souka AP, Nicolaides, KH. Diagnoses of fetal abnormalities at the 10-14 week scan. Ultrasound Obstet. Gynecol 1997, 10:429-442.

56. Small M, Copel JA. Indications for fetal echocardiography.2004; 25:210-222.

57. Smrcek JA, Berg C, Geipel A, Fimmers R, Diedrich K, Gembruch U. Early fetal echocardography: heart biometry and visualization of cardiac structures between 10 and 15 weeks gestation. J Ultrasound Med 2006; 25:173-182.

58. Trines J, Hornberger LK. Evolution of heart disease in utero. Pediatr Cardiol2004; 25:287-298.

59. Tworetzky W, Hang-Wilkins L, Jennings RW, van der Velde ME, Mashall AC, Marx GR, Colan SD, Benson CB, Lock JE, Perry SB. Congenital heart Disease. Circulation 2004; 110:2125-2131. 